\title{
The SMT Execution Service: Features, Fairness, and the Future
}

\author{
Morgan Deters \\ New York University, USA
}

\begin{abstract}
The SMT-Exec service is a benchmark repository, execution service, and competition infrastructure for the SMT community. Besides running the yearly competition and providing real-time (and also archival) results and analysis, SMT-Exec permits "private experiments" to be run year-round by researchers all over the world. These experiments are just like the yearly competition and run on the same computing cluster, but may be parameterized by users to run on benchmark and solver subsets of interest with a configurable timeout. Private solvers may be uploaded and tested against each other or against archival versions of competition solvers, and solvers and experiment results may be "published" so that they are publicly viewable. This talk describes SMT-Exec design highlights, challenges, growing pains, and current development plans for future versions of SMT-Exec.
\end{abstract}

